\title{
Chemical targeting of voltage sensitive dyes to specific cell types in the brain
}

Tomas Fiala1,„, Jihang Wang1,„, Matthew Dunn1, Peter Šebej¹,11, Ekeoma C. Nwadibia1, Diana M.

Martinez, ${ }^{4}$ Claire E. Cheetham ${ }^{6}$, Keri J. Fogle ${ }^{7,8}$, Michael J. Palladino ${ }^{7,8}$, Yuki Bando ${ }^{3,11}$, Rafael Yuste ${ }^{2,3}$, Zachary Freyberg ${ }^{9,10}$, David Sulzer ${ }^{4,5}$ and Dalibor Sames ${ }^{1,2 *}$

${ }^{1}$ Department of Chemistry, Columbia University, New York, NY, USA. ${ }^{2}$ NeuroTechnology Center at Columbia University, New York, NY, USA ${ }^{3}$ Department of Biological Sciences, Columbia University, New York, NY, USA. ${ }^{4}$ Departments of Psychiatry, Neurology and Pharmacology, Columbia University Medical Center, New York, NY, USA. ${ }^{5}$ Department of Molecular Therapeutics, New York Psychiatric Institute, New York, NY, USA. ${ }^{6}$ Department of Neurobiology, University of Pittsburgh, Pittsburgh, PA, USA. ${ }^{7}$ Department of Pharmacology \& Chemical Biology, University of Pittsburgh, Pittsburgh, PA, USA. ${ }^{8}$ Pittsburgh Institute of Neurodegenerative Diseases (PIND), University of Pittsburgh, Pittsburgh, PA, USA. ${ }^{9}$ Department of Psychiatry, University of Pittsburgh, Pittsburgh, PA, USA. ${ }^{10}$ Department of Cell Biology, University of Pittsburgh, Pittsburgh, PA, USA. ${ }^{11}$ Current addresses: RECETOX, Faculty of Science, Masaryk University, Brno, Czech Republic (P.Š), Department of Organ and Tissue Anatomy, Hamamatsu University School of Medicine, Hamamatsu, Shizuoka, Japan (Y.B.). *Correspondence should be addressed to D. Sames (ds584@columbia.edu) 


\section{Graphical abstract:}

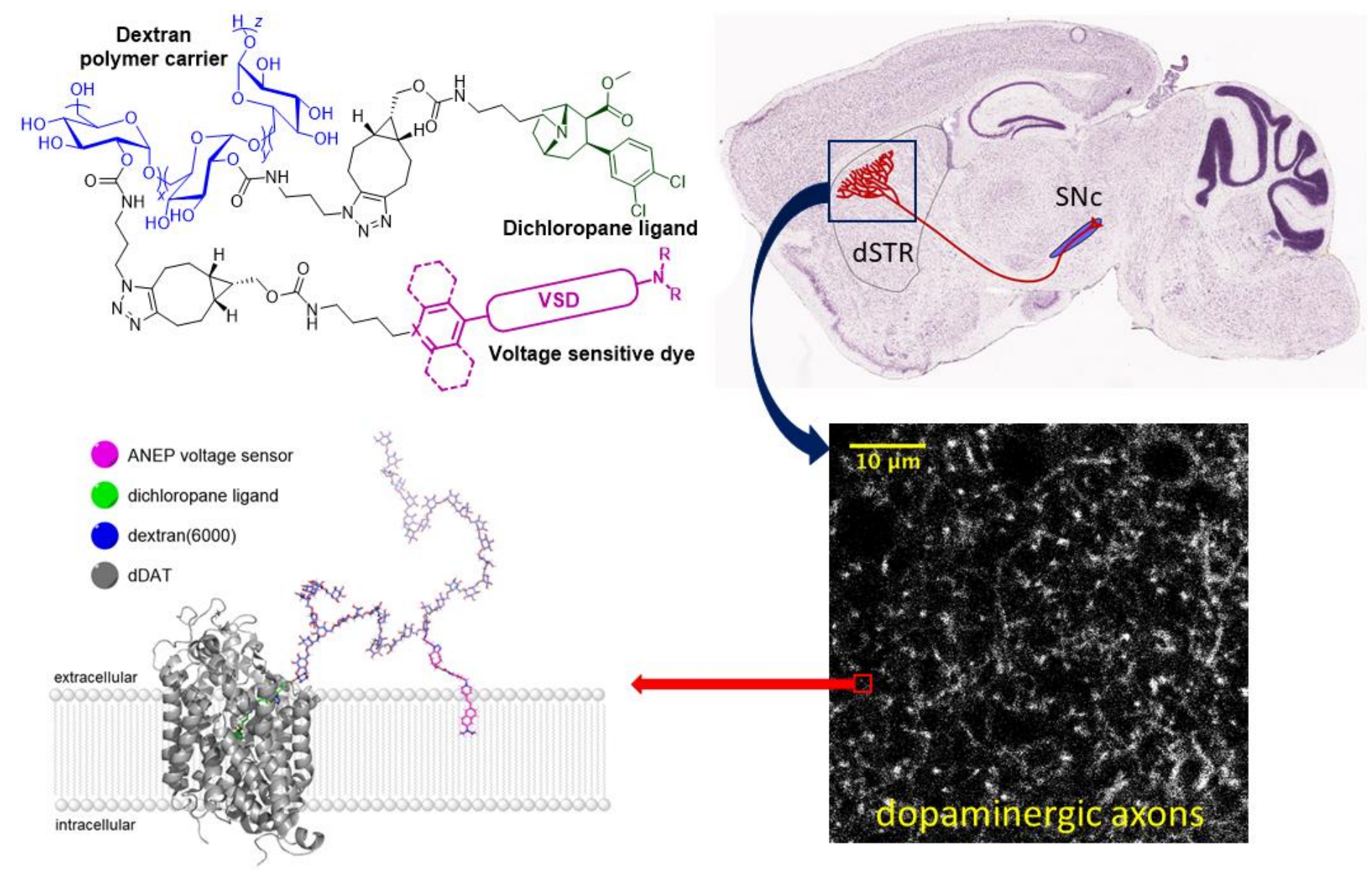




\section{Abstract}

Voltage sensitive fluorescent dyes (VSDs) are important tools for probing signal transduction in neurons and other excitable cells. These sensors, rendered highly lipophilic to anchor the conjugated $\pi$-wire molecular framework in the membrane, offer several favorable functional parameters including fast response kinetics and high sensitivity to membrane potential changes. The impact of VSDs has however been limited due to the lack of cell-specific targeting methods in brain tissue or living animals. We address this key challenge by introducing a non-genetic molecular platform for cell and molecule specific targeting of synthetic voltage sensitive dyes in the brain. We employ a dextran polymer particle to overcome the inherent lipophilicity of voltage sensitive dyes by dynamic encapsulation and target the construct to specific axonal extensions using the monoamine transporter ligand dichloropane. VoLDeMo (Voltage Sensor-Ligand-Dextran Targeted to Monoaminergic Neurons) probes label dense dopaminergic axons in the mouse striatum and sparse noradrenergic axons in the mouse cortex in acute brain slices. We also demonstrate in whole adult Drosophila brains that VoLDeMo targeting is ligand dependent. VoLDeMo variants bearing either a classical electrochromic ANEP dye or state-of-the-art VoltageFluor dye respond to membrane potential changes in a similar manner to the parent dyes, as demonstrated by whole-cell patch recording. The VoLDeMo platform enables targeting of diffusible VSD probes to specific neuronal cells using endogenous expression levels of native components of neurotransmission machinery. We envision that modularity of our platform will enable its application to a variety of molecular targets (other receptors and covalent labeling-based tags) and sensors (including those in other imaging modalities), as well as lipophilic drugs and signaling modulators. This work demonstrates the feasibility of a chemical targeting approach and expands the possibilities of cell-specific imaging and pharmacology. 


\section{Introduction}

Monitoring signal transmission between and within the cells of the nervous system has been and remains one of the key challenges in neuroscience. While patch-clamp electrophysiological techniques provided unprecedented insight into neuronal function and remain the standard technique to probe membrane potential dynamics in excitable cells, ${ }^{1,2}$ optical techniques have emerged as a powerful complement to electrode-based methods. The development of calcium sensitive dyes (CaSDs) $)^{3,4}$ and voltage sensitive dyes (VSDs) ${ }^{5-8}$ has transformed neuroscience and cell biology at large, however the key shortcoming of synthetic sensors has been the lack of targeting to specific cell types in brain tissue and in vivo. ${ }^{9-11}$ Consequently, the advancement of protein based calcium and voltage indicators have been intensely pursued, as these genetically encoded tools can be targeted to many neuronal cell types via cell-specific, promoter-based genetic methods. ${ }^{12-15}$

Synthetic dyes, however, have several key advantages over fluorescent proteins, mainly the availability of the entire chemical space for choice, design, and direct modification of the fluorophore, enabling tuning of photophysical and functional properties (e.g. absorption and emission profile, photostability, brightness, response magnitude $(\Delta F / F)$ and kinetics $),{ }^{8,16,17}$ as well as design of sensors for other imaging modalities, e.g. short wave infrared (SWIR) $)^{18,19}$ fluorescence or photoacoustic (PA) ${ }^{20-22}$ imaging.

Synthetic voltage sensors are however exceptionally challenging to deliver to selected cell types due to their inherent lipophilicity, which causes high background staining and limited diffusibility in tissue. Successful attempts to target synthetic VSDs to specific cell types have been scarce and have only been applied to cell culture based experimental systems. For example, a chemo-genetic approach taking advantage of cell-surface expression of alkaline phosphatase to dephosphorylate and thus induce membrane localization of an electrochromic aminonaphthyl-ethene-pyridinium (ANEP) VSD precursor was reported. ${ }^{23-25}$ A similar strategy was adopted recently using a porcine liver esterase to activate a fluorogenically silent precursor of VoltageFluor, a photoinduced electron transfer (PeT) based VSD. ${ }^{26}$ Similarly, a photoactivatable voltage sensor 
was reported which could be uncaged in a subset of cells by spatially precise illumination with blue light. ${ }^{27}$ However, none of these targeting strategies have been demonstrated in intact tissue preparations ex or in vivo.

We have pursued chemical targeting strategies to deliver synthetic fluorophores to specific neuronal cells and synaptic release sites in intact brain tissue, utilizing native components of the neurotransmission machinery. We developed fluorescent false neurotransmitters (FFNs), probes that act as substrates of relevant monoamine transporters, label dopaminergic or noradrenergic neurons and their synaptic vesicles, and enable optical recording of transmitter release at individual synaptic sites ex vivo and in vivo in rodents. ${ }^{28-33}$ For example, the probe FFN102 labels dopaminergic release sites in the mouse striatum with high selectivity, due to the selective transport of FFN102 by the dopamine transporter (DAT) into dopaminergic neurons. ${ }^{30}$ Encouraged by this success, and cognizant of specific endogenous expression of DAT in dopaminergic axons, we hypothesized that a high affinity DAT ligand would enable targeting dopaminergic axons in the brain with a variety of sensors and actuators, including lipophilic VSDs. The embodiment of this idea required the use and optimization of small carbohydrate polymers to accommodate the high lipophilicity of VSDs and serve as a molecular carrier for both the VSD and targeting ligand.

Here we report a chemical platform, which enables targeting of lipophilic VSDs to monoaminergic neurons in rodent and Drosophila brains. Our Voltage Sensor-Ligand-Dextran Targeted to Monoaminergic Neurons, or VoLDeMo, integrates three modalities into one multifunctional construct to achieve this goal. We selected two VSDs for this exploratory study: di-2-ANEPEQ (1), a well-established electrochromic hemicyanine VSD; ${ }^{34,35}$ and dsRVF5 (disulfo Rhodol VoltageFluor-5, 2), a disulfonated ${ }^{36}$ version of a state-of-the-art VSD (Fig. 1a). ${ }^{37}$ A second component, the tropane analog dichloropane $(3$, Fig. 1 b), is a potent ligand of dopamine and norepinephrine transporters (DAT and NET respectively) $)^{38,39}$ and enables not only cell-type specific but also molecule-specific targeting of the VoLDeMo probes. Both the VSD and the ligand are appended to a dextran polymer carrier (Fig. 1c), that compensates for the dye's lipophilicity and decreases non-specific labeling in cell cultures and in the mammalian brain. 
a

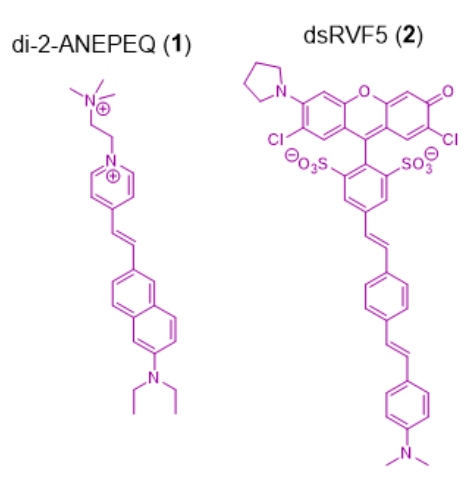

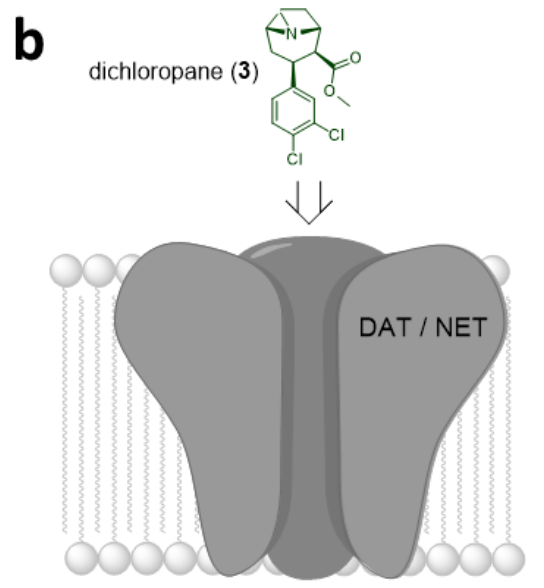

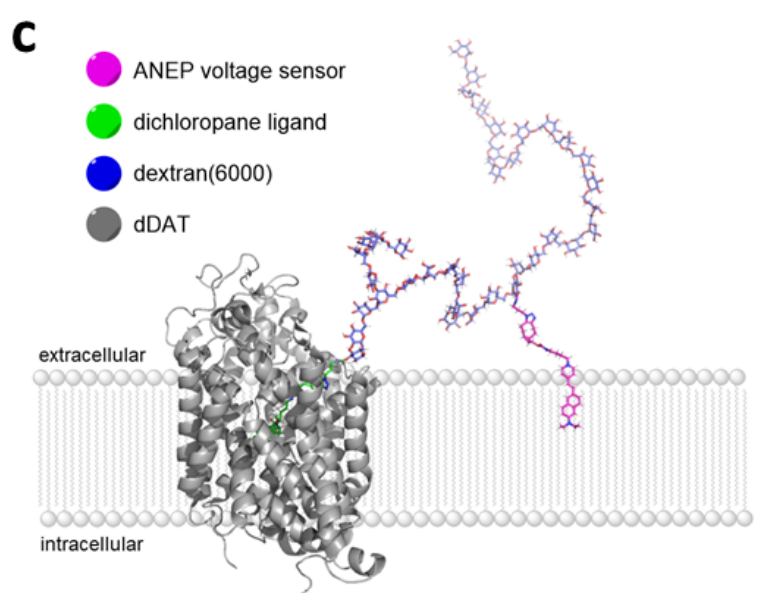

Fig. 1 | Components of the VoLDeMo targeting system. a, Voltage sensitive dyes di-2-ANEPEQ (1), ${ }^{34,35}$ and dsRVF5 (2). ${ }^{36,37} \mathbf{b}$, Dichloropane (3), a potent DAT and NET ligand. c, a 3D model of the VoLDeMo targeting system. A di-2-ANEP VSD (pink) and dichloropane ligand (green) are attached through short linkers to a dextran polymer carrier (blue). The dichloropane ligand of the construct is shown bound to the dopamine transporter (structure of drosophila $\mathrm{DAT}^{40}$ was used for the graphic), and the lipophilic VSD is inserted into the cell membrane.

\section{Results}

Establishment of dichloropane as a targeting ligand. Based on previous reports and examination of DAT's 3D structure, ${ }^{40,41}$ we hypothesized that introducing an $N$-Boc-4-aminobutyl linker instead of the methyl group on the bridgehead nitrogen of dichloropane (3) would largely conserve its affinity to monoamine transporters. Indeed, enantiomerically pure dichloropane analog 4 (Fig. 2a) blocked APP+ (an established DAT substrate ${ }^{42}$ ) uptake by HEK-293 cells stably transfected with human dopamine transporter (hDAT-HEK cells) with an $I_{50}$ of $11 \pm 1 \mathrm{nM}$ (Supplementary Fig. S6). We further synthesized rhodamine-labeled dichloropane (5, Fig. 2a), ${ }^{43}$ which strongly labeled hDAT-HEK cells in a coronal fashion, compared to null transfected cells, and the labeling was efficiently blocked by the DAT inhibitor, nomifensine ${ }^{44}$ (Supplementary Fig. S7). This system was employed as a positive control in subsequent VSD targeting studies. 


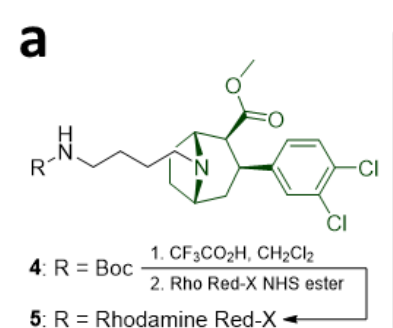

5: $\mathrm{R}=$ Rhodamine Red $-\mathrm{X}$

b

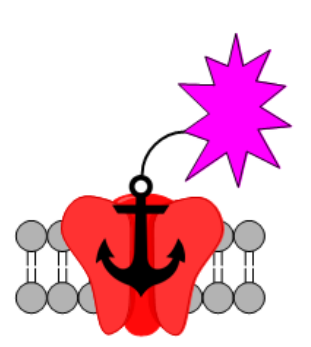

C
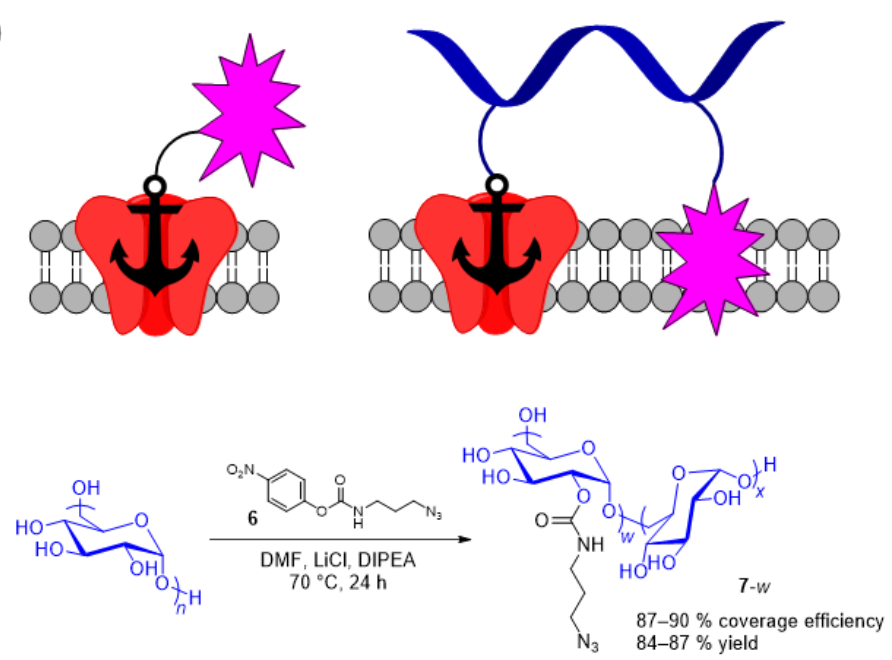

Rhodamine Red-X
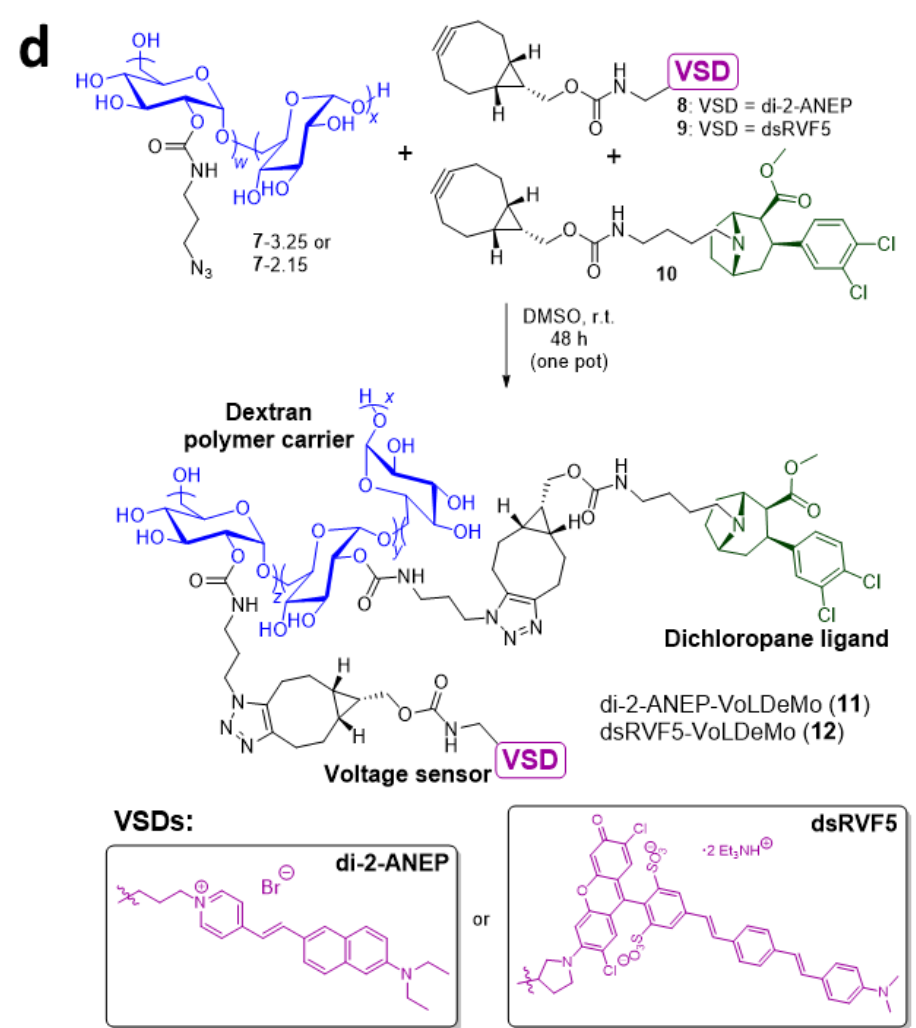

Fig. 2 | Design and synthesis of ligand guided fluorescent probes. a, Dichloropane ligand

bearing a Boc-4-aminobutyl linker (4) and its transformation into probe $\mathbf{5}$. b. Schematic illustration of ligand-dye fusion concepts. Left: a short linker separates the dye (pink) and ligand (black anchor) targeting a membrane protein of interest (red). Right: The dye and ligand are separated by a polymer carrier (blue) that enables insertion of the dye into the cell membrane (grey). c, Synthesis of azidopropyl-carbamate-dextran(6000) (7-w). Number $w$ reflects the degree of dextran functionalization with azidopropyl ligands (average number of azide functionalities per polymer molecule, see Supplementary Information for details). d, One-pot assembly of VoLDeMo via click chemistry by reacting $\mathrm{N}_{3}$-dextran $7-w(w=3.25$ or 2.15 , average number of azide functionalities per polymer molecule) with a 1:2 mixture of VSD 8 or $\mathbf{9}$ and ligand 10. Note: Dextran branching is omitted for clarity. Linker substitution on dextran is shown only in position 2; however, it is likely that positions 3, 4 and terminal 6 are also functionalized to a lower extent (see ref. ${ }^{45}$ and Supplementary Information). 


\section{Design, synthesis, and characterization of a non-genetic voltage sensitive dye-targeting platform.}

Targetable probe $\mathbf{5}$ is schematically illustrated in Fig. $2 b$-left. The same concept cannot be applied to VSD targeting, since a short linker would not enable insertion of the fluorophore into the cell membrane. A long linker and/or polymer carrier is needed to separate the ligand and the VSD (Fig. 2b-right) and render the construct functional. The first logical choice for a long ligand-VSD separator was a poly(ethylene glycol) (PEG) chain. PEG is a widely used polymer in chemical biology due to its solubility, synthetic accessibility, and presumed low protein binding. ${ }^{46}$ It has been successfully applied in wash-free labeling of plasma membrane proteins with the lipophilic Nile Red dye. ${ }^{47}$ We therefore constructed a dichloropane-PEG(3400)-di-2-ANEP construct (S71, Supplementary Scheme S8) and tested its targeting specificity in DAT-HEK cells. However, DAT-independent cell labeling, as well as internalization of construct S71 was observed (Supplementary Fig. S4). We thus concluded that PEG was unable to compensate for the lipophilic character of the VSD and render it targetable to specific membrane proteins.

We next turned our attention to dextran, a bacterial polysaccharide composed of glucopyranose units connected through $\alpha$-1,6-glycosidic linkages with occasional 1,3- and less frequently $1,2-$ and 1,4-branching. ${ }^{48}$ Dextran was an attractive choice due to its clinical use, biocompatibility, slow degradation in mammals, and high diffusion rates in tissue including brain. ${ }^{49-51}$ Based on our exploratory studies, we chose dextran(6000) $\left(M_{w}=6000 \mathrm{Da}\right)$ as a compromise between a large-enough hydrophilic surface to compensate for the lipophilicity of the VSD and a relatively small size for sufficient diffusion in tissue. We developed a functionalization protocol enabling attachment of 3-azidopropyl linker groups to dextran using activated carbamate 6 to provide azidopropyl-carbamate-dextran(6000) (7-w, Fig. 2c). This chemical labeling allowed for straightforward control of dextran coverage (average number of azide functionalities per polymer molecule $-w$; see Supplementary Information for detailed protocol and discussion of this parameter) with $\mathrm{N}_{3}$-linkers. Next, we used strain-promoted azide-alkyne cycloaddition click chemistry to attach functionalized di-2-ANEP (8) or dsRVF5 VSD (9) and dichloropane ligand (10). The position of the linker terminated with the clickable bicyclo[6.1.0]non-4-yn-9-yl)methyl (BCN) group was chosen based on synthetic feasibility and expected 
orientation of the VSD in the cell membrane. The ethylene-quaternary ammonium headgroup of di-2-ANEPEQ (1) was substituted with a flexible butylene linker terminated with carbamate-bound BCN in the functionalized dye 8 (linker attached via the pyridinium nitrogen). Similarly, dsRVF5 9 was functionalized with BCN at the pyrrolidine group, which faces the extracellular space upon membrane localization of the VSD. The sensors and ligand equipped with the strained alkyne moiety were reacted with the azide functionalized dextran 7-w, assembling the final di-2-ANEP-VoLDeMo (11) and dsRVF5-VoLDeMo (12) constructs respectively (Fig. 2d). The coverage of the dextran polymer with ligands $(y)$ and VSDs $(z)$ was chosen as follows: to minimize the number of polymer molecules which would carry 2 or more lipophilic VSDs (thus increasing the probability of non-specific binding), we aimed for $y<1$; and to ensure that a vast majority of polymer molecules that carry a VSD also carry at least 1 dichloropane ligand, we aimed for $z>1$ and $y: z=1: 2$. di-2-ANEP-VoLDeMo was obtained with a coverage of 0.74 ANEP/polymer and 1.38 ligand/polymer; similarly, dsRVF5-VoLDeMo was obtained with a comparable coverage of $0.64 \mathrm{dsRVF5/polymer}$ and 1.33 ligand/polymer as determined by ${ }^{1} \mathrm{H}$ NMR (Supplementary Fig. S2 and S3). The concentration values in subsequent experiments are calculated based on average molecular weights of the constructs (see Supplementary Information for details); therefore, $100 \mathrm{nM}$ VoLDeMo probe contains $\sim 74$ nM di-2-ANEP VSD or $\sim 64$ nM dsRVF5 VSD.

A significant difference was observed between the ${ }^{1} \mathrm{H}$ NMR spectra of VoLDeMo probes in $\mathrm{D}_{2} \mathrm{O}$ and DMSO- $d_{6}$. While broad signals were observed in the aromatic region in $\mathrm{D}_{2} \mathrm{O}($ Fig. $3 a)$, much sharper peaks were observed in DMSO-d6 (Fig. 3b). This observation provides a clue about the conformational behavior of these constructs: the structure is likely folded in aqueous environments, wherein the lipophilic dye is concealed by the dextran polymer to minimize the unfavorable VSD-water interactions. Restricted flexibility of the VSD in such a folded state results in broadened peaks. Signals belonging to the dichlorophenyl group of the dichloropane ligand show significantly less broadening (green asterisks in Fig. 3a) reflecting less shielding by dextran. Only in the presence of a less polar (and aprotic dipolar) environment (DMSO- $d_{6}$ ) will the VoLDeMo probe favor an unfolded conformation. We therefore propose the following targeting model for the VoLDeMo sensors: in the bulk extracellular environment, the construct exists predominantly in a folded state with the VSD 
minimally exposed to the aqueous environment, which results in low non-specific binding. Association of the dichloropane ligand with DAT or NET increases the effective concentration of the VoLDeMo construct at the cell membrane, the conformational equilibrium is shifted, and the VSD partitions into the membrane (Fig. 3c).

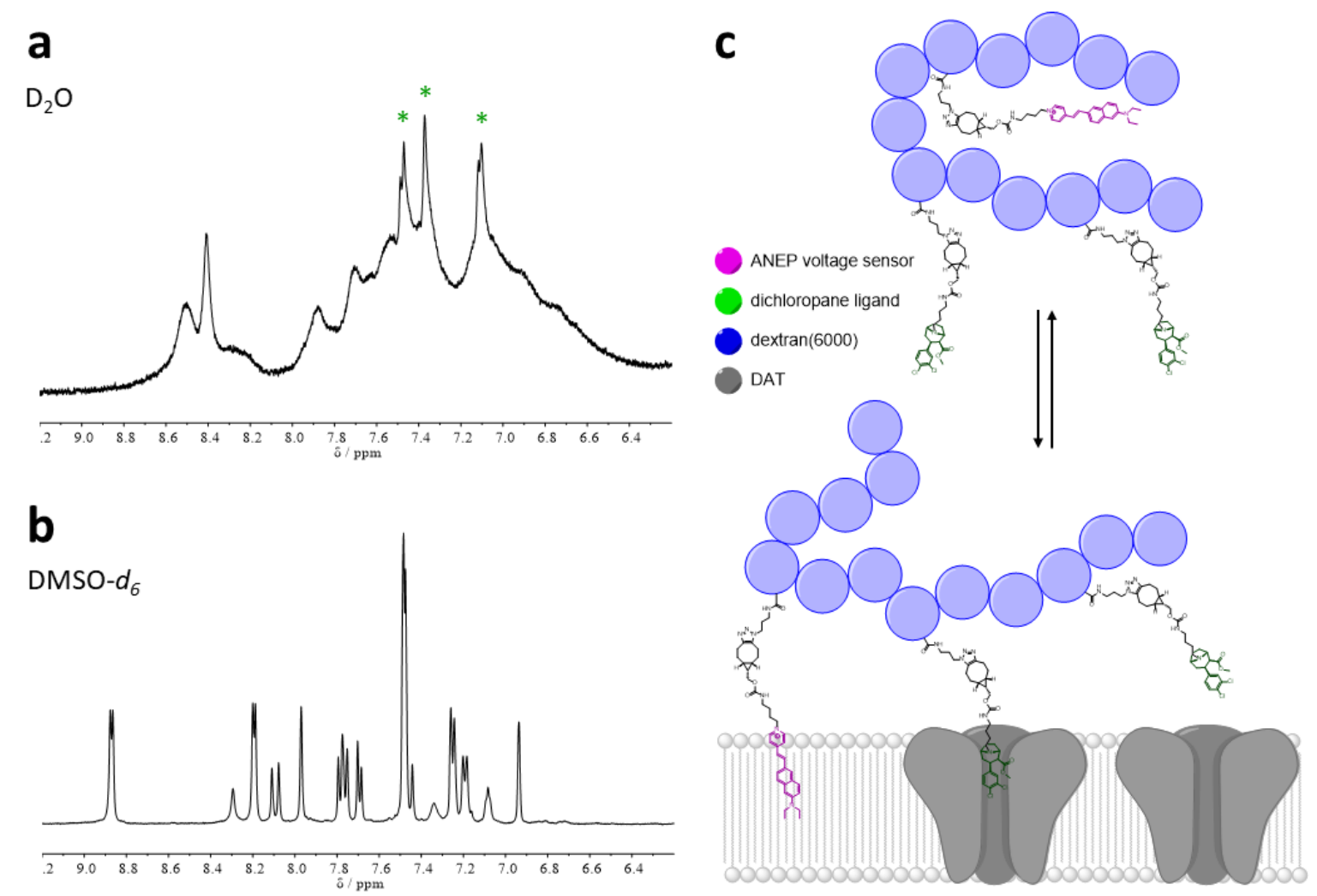

Fig. 3 | Proposed conformational behavior and targeting model of VoLDeMo. a, ${ }^{1} \mathrm{H}$ NMR

(500 MHz, $\mathrm{D}_{2} \mathrm{O}$ ), zoomed in aromatic region (6.2-9.2 ppm) of di-2-ANEP-VoLDeMo. Broadened peaks reflect restrained conformational freedom of the lipophilic VSD which is shielded by the dextran carrier. Green asterisks - signals of the dichloropane ligand which are significantly sharper, reflecting more conformational freedom of the ligand. b, ${ }^{1} \mathrm{H}$ NMR (500 MHz, DMSO-d6), zoomed in aromatic region (6.2-9.2 ppm) of di-2-ANEP-VoLDeMo. Sharp peaks indicate high conformational flexibility of the construct in the more solubilizing environment of DMSO.

c, Targeting model of VoLDeMo: Top - in bulk aqueous environment, the VSD (pink) is encapsulated by the dextran carrier (blue spheres) from the solvent. Bottom - after binding of the dichloropane ligand (green) to DAT (grey), the equilibrium is shifted and the VSD partitions into the cell membrane. 
VoLDeMo selectively labels DAT-expressing cells and enables optical recording of membrane potential

changes. hDAT-HEK cells were incubated with di-2-ANEP-VoLDeMo or dsRVF5-VoLDeMo (100 nM) for 5 min or 15 min respectively at room temperature and washed with DPBS, resulting in membrane-localized fluorescence staining. Upon pre- and co-treatment with the DAT inhibitor, nomifensine $(2 \mu \mathrm{M})$, the membrane staining of hDAT-transfected cells was significantly diminished $(20.2 \pm 5.7$ fold decrease, $n=3, P=0.0013$ for di-2-ANEP-VoLDeMo and $3.4 \pm 0.5$ fold decrease, $n=3, P<0.0001$ for dsRVF5-VoLDeMo, two-tailed unpaired $t$-test). Additionally, coronal staining was not observed with null-transfected HEK cells under identical conditions $(8.4 \pm 1.5$ fold fluorescence decrease with respect to hDAT-HEK cells, $n=3, P=0.0018$ for di-2-ANEP-VoLDeMo and $6.0 \pm 0.6$ fold, $n=3, P<0.0001$ for dsRVF5-VoLDeMo, two-tailed unpaired $t$-test), confirming that binding to DAT is required for localization of the probe to the surface of the membrane (Fig. $4 a$ and $d$, Supplementary Fig. S8). Higher signal to background observed for di-2-ANEP-VoLDeMo compared to dsRVF5-VoLDeMo is likely a result of the lower lipophilicity of the di-2-ANEP VSD with respect to dsRVF5, which results in a lower extent of non-specific background staining. 
a

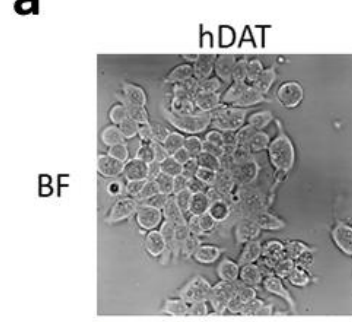

di-2-ANEP-VoLDeMo

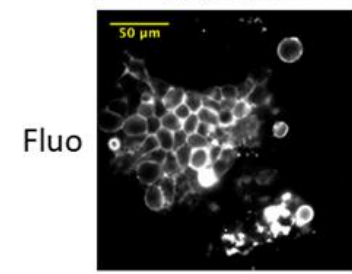

b

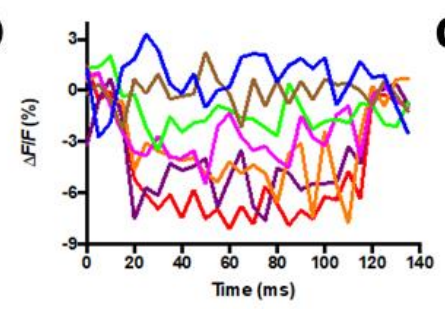

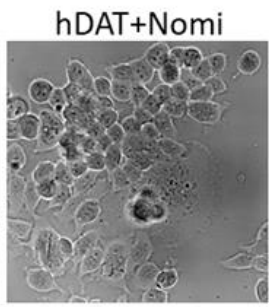

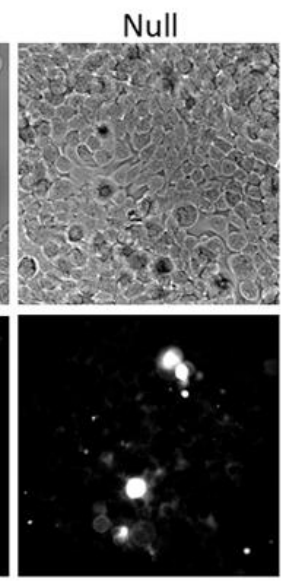

C

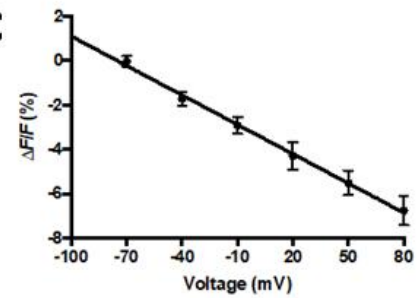

d

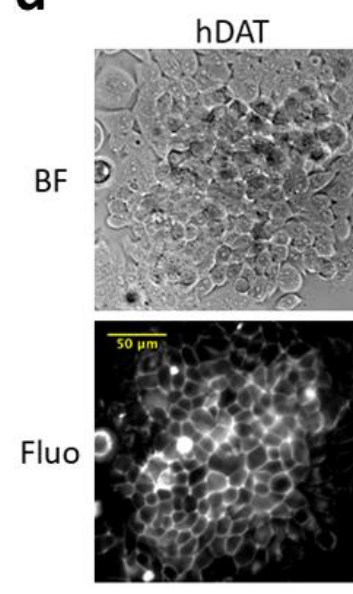

e

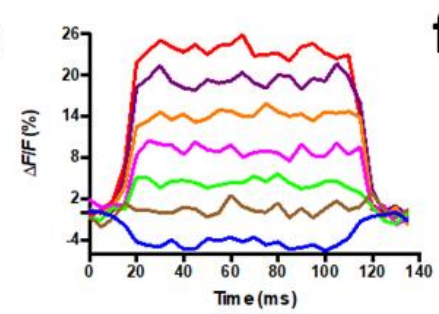

dsRVF5-VoLDeMo

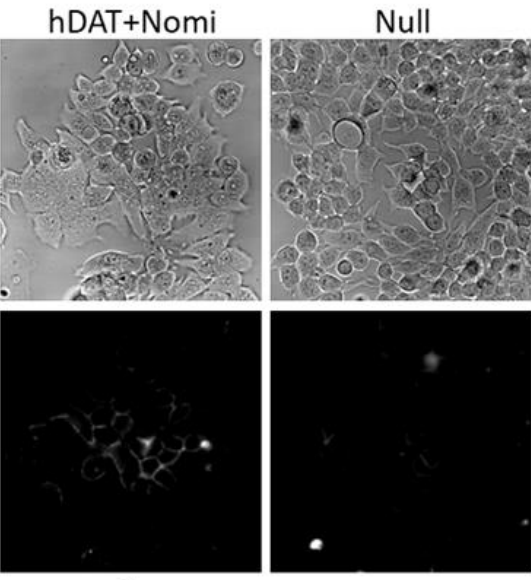

f

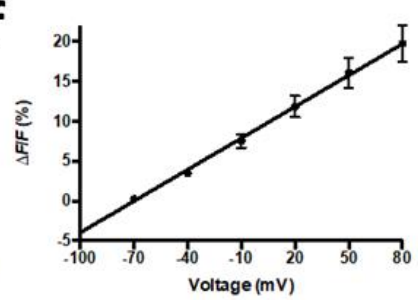

Fig. 4 | Labeling specificity and membrane potential sensitivity of VoLDeMo probes in hDAT-

HEK cells. a, d, Brightfield (BF, top) and corresponding fluorescence (Fluo, bottom) images of HEK cells labeled with di-2-ANEP- (a) and dsRVF5-VoLDeMo (d) probes (100 nM). Labeling was compared in hDAT-HEK cells (left), hDAT-HEK cells pre-incubated with the DAT inhibitor nomifensine ( $2 \mu \mathrm{M} ;$ middle), and null-transfected HEK cells (right). All fluorescence images are equally contrasted for each VoLDeMo probe. b, e, Representative concatenated traces of the fractional change in fluorescence of di-2-ANEP- (b) and dsRVF5-VoLDeMo (e) probes (100 nM) versus time of a voltage-clamped $\mathrm{HEK}$ cell, held at $-60 \mathrm{mV}$ and then subjected to $100 \mathrm{~ms}$ voltage holdings ranging from $-100 \mathrm{mV}$ (blue trace) to $+80 \mathrm{mV}$ (red trace) in $30 \mathrm{mV}$ intervals. c, f, Plots of percent fluorescence change of di-2-ANEP- (c) and dsRVF5-VoLDeMo (f) probes (100 nM) versus membrane voltage. $\Delta F / F$ per $100 \mathrm{mV}=-4.7 \% \pm 1.1 \%, S N R=4.5 \pm 0.5, n=5$; mean \pm SEM for di2-ANEP-VoLDeMo. $\Delta F / F$ per $100 \mathrm{mV}=13.1 \% \pm 3.7 \%, S N R=14.2 \pm 2.9, n=5$; mean $\pm \mathrm{SEM}$ for dsRVF5-VoLDeMo.

We further verified that di-2-ANEP and dsRVF5 dyes remain voltage sensitive when attached to dextran as a part of VoLDeMo probes. hDAT-HEK cells labeled with di-2-ANEP-VoLDeMo or dsRVF5-VoLDeMo 
(100 nM) were whole-cell voltage clamped. The cells were first held at $-60 \mathrm{mV}$ to mimic the resting membrane potential of neurons, then subjected to DC holding potentials steps ranging from $-100 \mathrm{mV}$ to $+80 \mathrm{mV}$ in $30 \mathrm{mV}$ intervals and imaged at $200 \mathrm{~Hz}$ (Fig. $4 b$ and $e$ ). In the case of di-2-ANEP-VoLDeMo, we observed a decrease in fluorescence intensity following depolarization of the membrane potential, which is common for electrochromic VSDs when excited at the red edge of their absorption band. ${ }^{52}$ When the fractional fluorescence change was plotted against the membrane potential, a linear plot $\left(R^{2}=0.998\right)$ with a $\Delta F / F$ per $100 \mathrm{mV}=-4.7 \% \pm 1.1 \%$ was obtained ( $n=5$, Fig. $4 c$ ). This result was comparable to what was previously demonstrated for ANEP class dyes. ${ }^{52}$ For dsRVF5-VoLDeMo, in contrast, we observed increased fluorescence intensity with increasing membrane potential, as expected for the PeT-based class of VSDs. ${ }^{37}$ The plot of fractional fluorescence change vs. membrane potential was linear $\left(R^{2}=0.999\right)$ with a $\Delta F / F$ per $100 \mathrm{mV}=13.1 \% \pm 3.7 \%(n=5$, Fig. 4f).

Under whole-cell voltage clamp mode, hDAT-HEK cells were also subjected to simulated trains of rapid depolarizations to explore the temporal resolution of VoLDeMo probes in resolving AC voltage changes. Cells labeled with di-2-ANEP-VoLDeMo or dsRVF5-VoLDeMo (100 nM) were held at $-60 \mathrm{mV}$ and subjected to bursts of ten individual voltage spikes of $2 \mathrm{~ms}$ length and $100 \mathrm{mV}$ magnitude to resemble the membrane action potential of a typical neuron. The ten voltage spikes within one burst were applied at $100 \mathrm{~Hz}$ in frequency. At $1 \mathrm{kHz}$ imaging rate, both VoLDeMo probes were able to detect and distinctively resolve individual voltage spikes within one burst (Supplementary Fig. S9).

VoLDeMo probes deliver VSDs to dopamine and norepinephrine axons in mouse brain tissue. The next key test for the targeted constructs was the examination of labeling specificity in acute mouse brain tissue, specifically in two regions with vastly different density of catecholamine axons, the dorsal striatum and somatosensory cortex (Fig. 5). The dorsal striatum (dSTR) receives dense dopaminergic axonal projections from neurons originating in the substantia nigra compacta $(\mathrm{SNc})$ in the midbrain, that highly arborize at their target (Fig. 5a). ${ }^{53}$ Striatal brain slices (living slice preparation, see Supplementary Information) incubated with di-2-ANEP-VoLDeMo (100 nM, $30 \mathrm{~min}$ ) exhibited a string-like, punctate pattern of fluorescent labeling 
(Fig. 5b, Supplementary Fig. S10a), typical for dopaminergic axons in this region. Quantitative colocalization with an established dopamine probe FFN102 gave a high level of colocalization $(78.0 \% \pm 3.51 \%, n=3$, Supplementary Fig. S11), which was significantly higher than the respective scrambled control $(17.3 \% \pm 2.33 \%, P<0.0001$, two-tailed unpaired $t$-test $)$. The pattern of the label was similar to that previously reported in the striatum of TH-GFP mice, where GFP is expressed under the control of tyrosine hydroxylase (TH) promoter, a marker of catecholamine neurons, and that obtained via DAT immunolabel. ${ }^{30}$ As expected, pre-incubation and co-incubation with nomifensine $(2 \mu \mathrm{M})$ diminished the targeting of dopamine axons and resulted in predominantly diffuse background labeling, indicating that the axonal labeling is dependent on DAT. To further demonstrate the high degree of VoLDeMo selectivity, we incubated striatal brain slices with untethered di-2-ANEPEQ (1, $100 \mathrm{nM}, 30 \mathrm{~min})$. As expected, a dramatically contrasting pattern corresponding to non-specific fluorescent labeling of cell membranes was observed (Fig. 5b). To our knowledge, this is the first example of selective delivery of diffusible VSDs to specific neuronal cell types in mammalian brain tissue, as well as the first direct targeting to specific axonal projections and release sites.

We next tested labeling of striatal slices with dsRVF5-VoLDeMo bearing the more lipophilic and larger rhodol based VSD. Remarkably, STRd brain slices incubated with dsRVF5-VoLDeMo (100 nM, 30 min) exhibited a similar string-like pattern of fluorescent labeling as its di-2-ANEP counterpart (Fig. 5c, Supplementary Fig. S1Ob). Pre-incubation and co-incubation with nomifensine $(2 \mu \mathrm{M})$ produced a diffuse background staining (Fig. 5c). The high degree of dsRVF5-VoLDeMo selectivity in striatal slice was further demonstrated by comparison to untethered dsRVF5 $(2,100 \mathrm{nM}, 30 \mathrm{~min}$, Fig. 5c), which labels many cell membrane compartments. 

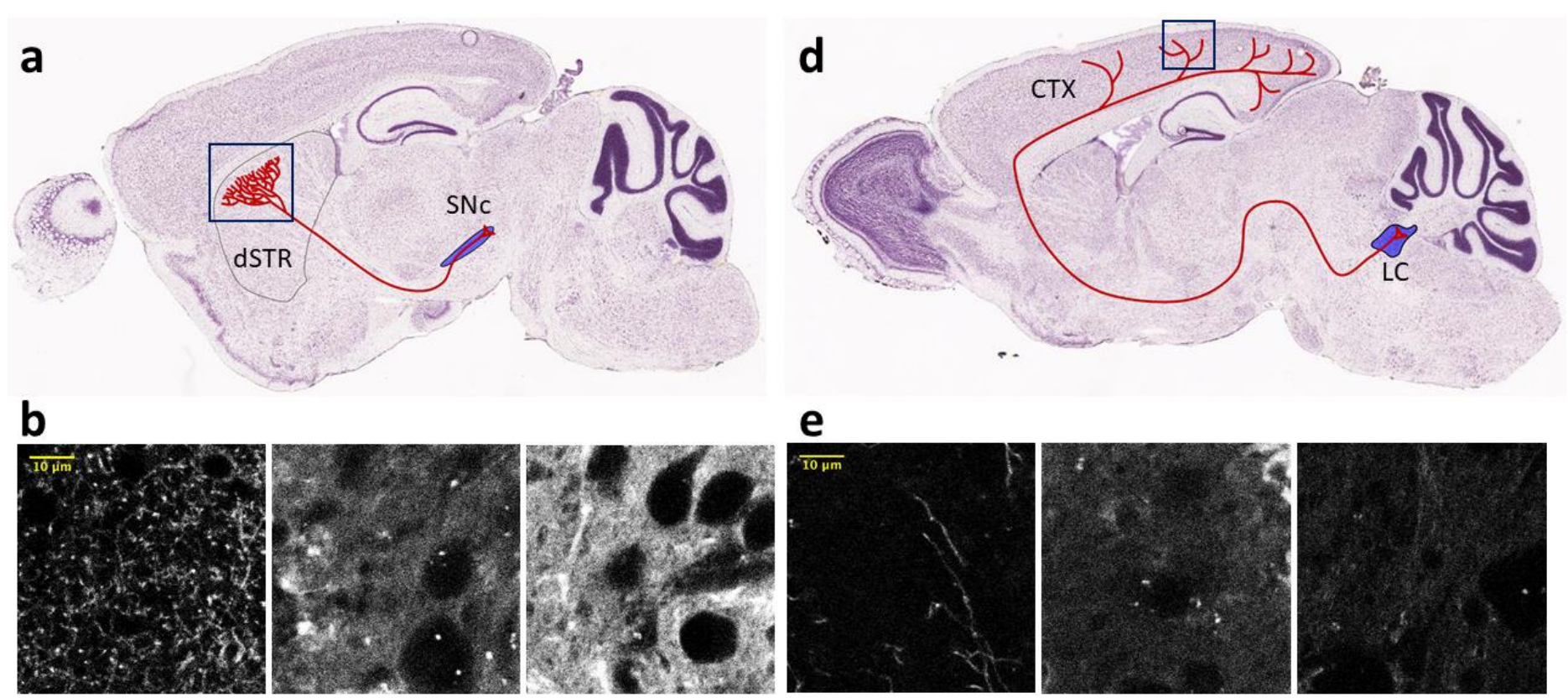

di-2-ANEP-VoLDeMo

+ nomifensine

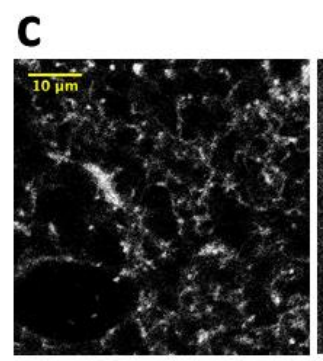

dsRVF5-VoLDeMo

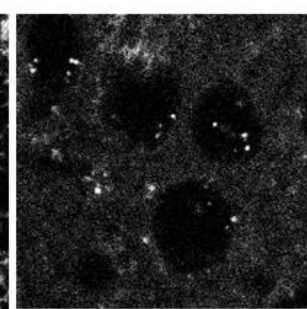

+ nomifensine
di-2-ANEPEQ (1)

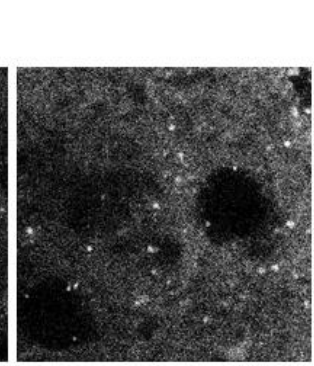

dsRVF5 (2)

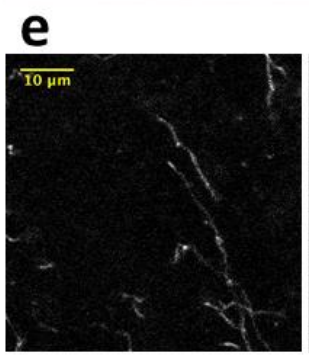

di-2-ANEP-VoLDeMo

f

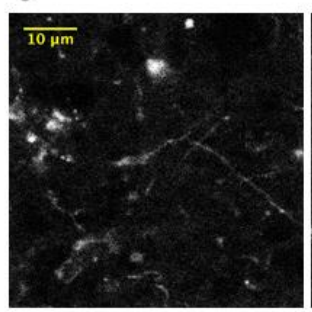

dsRVF5-VoLDeMo

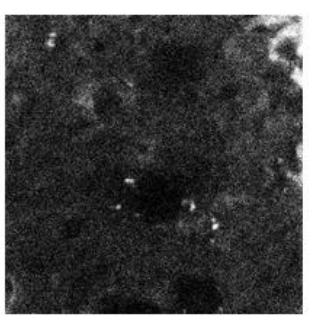

+ nomifensine

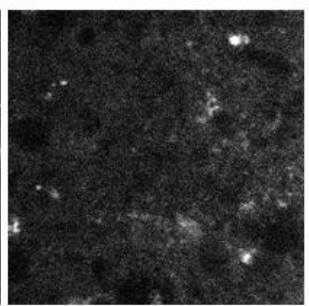

+ nomifensine

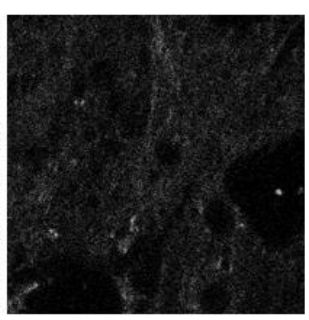

di-2-ANEPEQ (1)

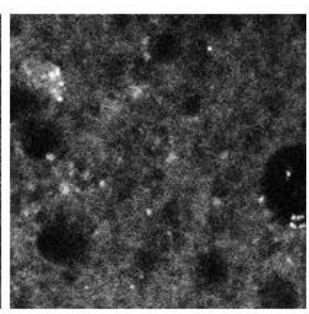

dsRVF5 (2)

Fig. 5 | Targeting of VoLDeMo probes to catecholamine axons in mouse brain slices. a, Nissl

stained sagittal slice of a mouse brain (image credit: Allen Institute ${ }^{54,55}$ ) with a schematic

representation of a dopaminergic neuron originating in the SNc and highly arborizing in the dSTR.

The blue rectangle indicates the approximate region where the images in $\mathbf{b}$ and $\mathbf{c}$ were acquired via two-photon microscopy. b, c, Representative images of striatal slice labeling with di-2-ANEP and dsRVF5 probes, respectively. Left: striatal slice incubated with the targeted di-2-ANEP-VoLDeMo (100 nM, $30 \mathrm{~min}, 15 \mu \mathrm{m}$ depth from the tissue surface) or dsRVF5-VoLDeMo (100 nM, $30 \mathrm{~min}$, $15 \mu \mathrm{m}$ depth) respectively; middle: an analogous experiment with pre- and co-incubation with the DAT/NET blocker nomifensine $(2 \mu \mathrm{m})$; right: striatal slice incubated with untargeted di-2-ANEPEQ (1, $100 \mathrm{nM}, 30 \mathrm{~min}, 15 \mu \mathrm{m}$ depth) or dsRVF5 (2, $100 \mathrm{nM}, 30 \mathrm{~min}, 15 \mu \mathrm{m}$ depth). d, Nissl stained sagittal slice of a mouse brain (image credit: Allen Institute ${ }^{54,55}$ ) with a schematic 
representation of a noradrenergic neuron originating in the LC with sparse axonal projections in the CTX. The blue rectangle indicates the approximate region in the somatosensory cortex where the images in $\mathbf{e}$ and $\mathbf{f}$ were acquired via two-photon microscopy. $\mathbf{e}, \mathbf{f}$, Representative images of cortical slice labeling with di-2-ANEP and dsRVF5 probes, respectively. Left: cortical slice incubated with the targeted di-2-ANEP-VoLDeMo (100 nM, $30 \mathrm{~min}, 15 \mu \mathrm{m}$ depth) or dsRVF5-VoLDeMo (100 nM, $30 \mathrm{~min}, 15 \mu \mathrm{m}$ depth), respectively; middle: an analogous experiment with pre- and co-incubation with nomifensine $(2 \mu \mathrm{M})$; right: striatal slice incubated with untargeted di-2-ANEPEQ (1, $100 \mathrm{nM}$, $30 \mathrm{~min}, 15 \mu \mathrm{m}$ depth) or dsRVF5 $(2,100 \mathrm{nM}, 30 \mathrm{~min}, 15 \mu \mathrm{m}$ depth). Images labeled by the same letter were contrasted equally. ANEP probes were excited at $920 \mathrm{~nm}$ and collected at 570-610 nm. dsRVF5 probes were excited at $820 \mathrm{~nm}$ and collected at 570-610 $\mathrm{nm}$.

The somatosensory cortex (CTX), unlike the dSTR, contains a far lower density of catecholaminergic axons, which we recently reported are mostly noradrenergic extensions expressing $\mathrm{NET}^{32}$ originating in the locus coeruleus (LC) (Fig. 5d). ${ }^{56}$ These sparse projections represent a far more challenging target for VoLDeMo probes, due to the greatly lower ratio of target noradrenergic cells to other non-target cells and tissue components. Despite this, cortical brain slices incubated with di-2-ANEP-VoLDeMo (100 nM, $30 \mathrm{~min})$ exhibited a sparse fluorescent labeling pattern (Fig. 5e, Supplementary Fig. S10c) typical of noradrenergic axons in this brain area. ${ }^{32}$ There appeared to be no prominent non-specific uptake of the probes by resident cell bodies, either in the cortex or striatum. Similar control experiments were employed as in the striatal slice: preincubation and co-incubation with nomifensine $(2 \mu \mathrm{M})$ nearly abolished the string labeling (Fig. 5e); and incubation of a cortical brain slice with untethered di-2-ANEPEQ (1, $100 \mathrm{nM}, 30 \mathrm{~min})$ produced a pattern corresponding to non-specific fluorescent labeling of the brain tissue (Fig. 5e). Consistent with appropriate targeting, dsRVF5-VoLDeMo (100 nM, $30 \mathrm{~min})$ produced a sparse string-like fluorescent labeling pattern in the cortical slice (Fig. 5f, Supplementary Fig. S10d), albeit with higher background than di-2-ANEP-VoLDeMo (Fig. 5e). This construct also showed occasional bright punctate or tubular objects larger than the axonal strings or varicosities, which we ascribe to non-specific labeling and/or clustering of the probe. While the combination 
of a low density of noradrenergic projections in somatosensory cortex together with the higher lipophilicity of the dsRVF5 VSD tested the limits of the VoLDeMo delivery system, a dramatic contrast was observed compared to images obtained with nomifensine $(2 \mu \mathrm{M})$ inhibition and unmodified dsRVF5 (2, $100 \mathrm{nM}, 30 \mathrm{~min}$, Fig. 5f).

Both di-2-ANEP- and dsRVF5-VoLDeMo constructs showed good diffusion in living tissue, capable of carrying the embodied lipophilic VSDs to depths typically used in our laboratories in acute slice under the standard experimental conditions (100 nM probe concentration, 30 min incubation time, washing, and common instrumental settings). The string-like labeling pattern remained distinct up to $30 \mu \mathrm{m}$ below surface in the striatum and $100 \mu \mathrm{m}$ below surface in the cortex. The probes showed less penetration in dSTR, presumably due to the high density of dopaminergic projections, which erects an additional diffusion barrier by leaving less material available for diffusion further from the surface (referred to as the "affinity barrier" in the antibody field $\left.{ }^{57}\right)$. In addition, the less lipophilic di-2-ANEP-VoLDeMo generally penetrated deeper than dsRVF5-VoLDeMo under the same conditions.

Targeting of VoLDeMo probes in whole Drosophila brains. The brain of the adult fruit fly (Drosophila melanogaster $)$ is small ( $200 \mu \mathrm{m}$ in depth). Combined with the genetic tractability, this organism serves as a useful model for studying intact circuitry in the whole brain via multiphoton microscopy. ${ }^{58-60}$ Therefore, we tested targeting of VoLDeMos in whole fly brains. We used adult brains from Drosophila melanogaster expressing the GEVI ArcLight ${ }^{60}$ under the TH promotor and focused on the suboesophageal ganglion (SOG) region, due to its easy accessibility for multiphoton imaging and high density of dopamine axonal varicosities. Incubating a TH-driven ArcLight fly brain with di-2-ANEP-VoLDeMo (100 nM, 30 min) resulted in sparse punctate staining with partial overlap with ArcLight $(n=4$ brains, Fig. $6 a)$, which was abolished by pre- and co-incubation with excess competing ligand, dichloropane 4 ( $2 \mu \mathrm{M}, 30$ min pre-incubation, $n=3$ brains $)$, leaving only a diffuse labeling pattern consistent with background fluorescence. Incubation of the brains with untethered di-2-ANEPEQ (1, $100 \mathrm{nM}, 30 \mathrm{~min})$ resulted in similar non-punctate, non-specific labeling $(n=3$ 
brains, Fig. 6c), thus highlighting the labeling selectivity of the VoLDeMo probe, and indicating that the labeling is ligand-dependent. While there is partial co-localization of VoLDeMo with TH-driven ArcLight, which suggests co-localization of the two fluorescent signals to dopamine nerve varicosities, we also observed VoLDeMo puncta that were not co-labeled by Arclight. Given that the VoLDeMo probe labels other monoaminergic transporters including the serotonin transporter, it is possible that this additional labeling is due to association with these related monoamine transporters in Drosophila. ${ }^{61,62}$ Lastly, incubating a TH-driven ArcLight-expressing Drosophila brain with dsRVF5-VoLDeMo (100 nM, $30 \mathrm{~min}$ ) resulted in a similar punctate staining pattern as for the di-2-ANEP targeted probe ( $n=3$ brains, Fig. $6 d)$. 


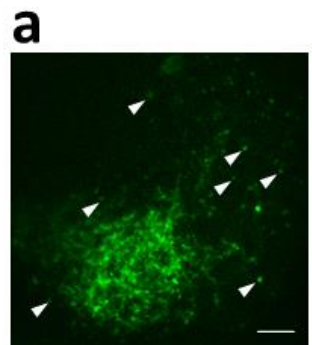

ArcLight

b

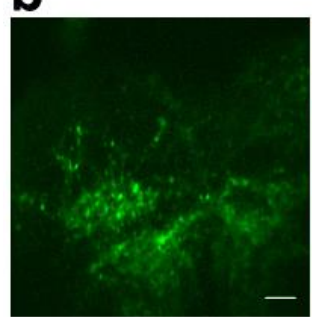

ArcLight

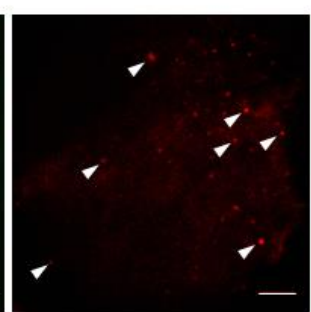

di-2-ANEP-VoLDeMo

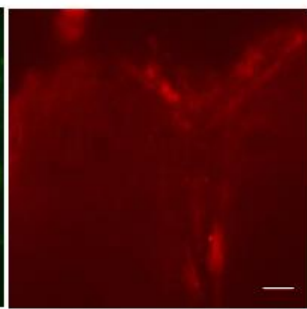

di-2-ANEP-VoLDeMo+4

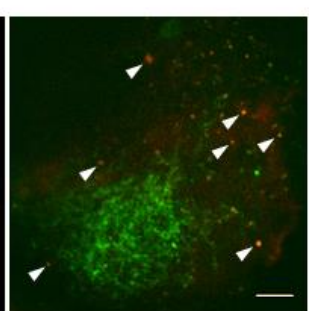

merge

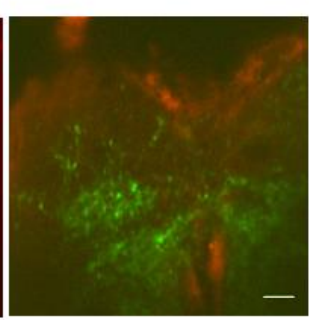

merge

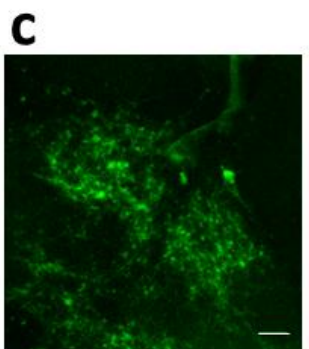

ArcLight

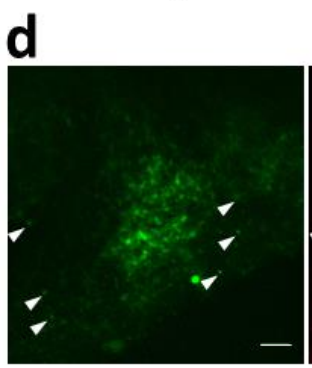

ArcLight

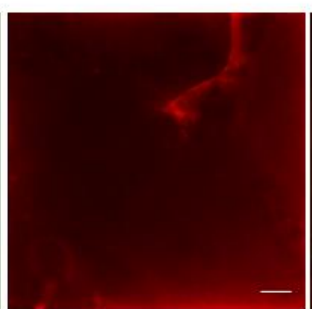

di-2-ANEPEQ (1)

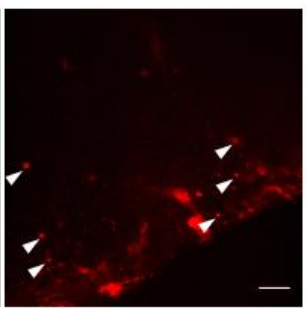

dsRVF5-VoLDeMo

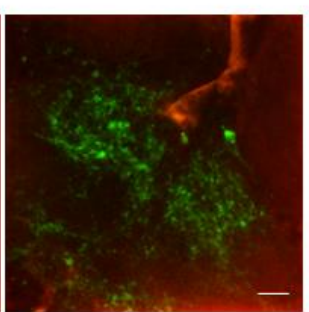

merge

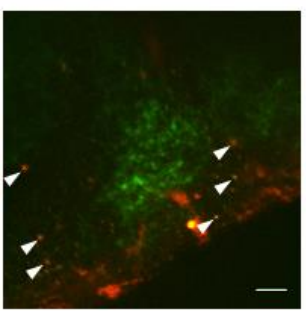

merge

Fig. 6 | Targeting of VoLDeMo probes in whole Drosophila brains. All panels: Excitation wavelength: $920 \mathrm{~nm}$. ArcLight (left); the respective synthetic VSD (middle); merged left and middle images (right). All images are zoomed in to the suboesophageal ganglion (SOG). The scale bars are $10 \mu \mathrm{m} . \mathbf{a}, \mathrm{A}$ TH-driven ArcLight-expressing fly brain incubated with di-2-ANEP-VoLDeMo (100 nM, $30 \mathrm{~min}$ ) resulted in punctate staining with partial overlap with ArcLight-labeled dopamine axonal varicosities (representative image; $n=4$ brains). Examples of co-localizing puncta are highlighted with white arrowheads. b, A TH-driven ArcLight fly brain incubated with di-2-ANEP-VoLDeMo (100 nM, $30 \mathrm{~min})$ and pre- and co-incubated with dichloropane 4 (targeting ligand of the construct, $2 \mu \mathrm{M}, 30 \mathrm{~min}$ pre-incubation) as a competing ligand resulted only in background staining without any punctate character or overlap with ArcLight localized to dopamine terminals (representative image; $n=3$ brains). c, A TH-driven ArcLight fly brain incubated with untethered di-2-ANEPEQ $(1,100 \mathrm{nM}, 30 \mathrm{~min})$ resulted in non-specific staining without any punctate character or overlap with ArcLight (representative image; $n=3$ brains). d, A TH-driven ArcLight fly brain incubated with dsRVF5-VoLDeMo (100 nM, $30 \mathrm{~min}$ ) resulted in punctate staining with partial overlap with ArcLight (representative image; $n=3$ brains). Examples of co-localizing puncta are highlighted with white arrowheads. 


\section{Discussion}

We have developed a targeting platform that enables delivery of lipophilic voltage sensitive dyes to specific neuronal cell types in brain tissue. This system consists of three components combined into one macromolecular sensor: 1) A fluorescent indicator of membrane potential (lipophilic voltage sensitive dye). The classical electrochromic di-2-ANEP ${ }^{34,35}$ and state-of-the-art dsRVF5 ${ }^{36,37}$ probes were chosen as model VSDs; 2) a dichloropane ligand that binds with high affinity to the family of monoamine transporter membrane proteins from the extracellular side; ${ }^{38,39} 3$ ) a polar polymer carrier represented by the polysaccharide dextran that compensates for the lipophilicity of the VSD by dynamic encapsulation, and so limits non-specific binding of

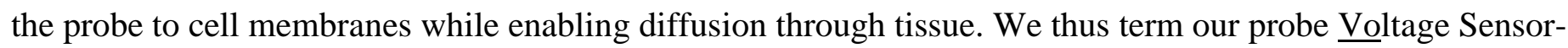
Ligand-Dextran Targeted to Monoaminergic Neurons, or VoLDeMo.

In contrast to previous approaches, which were successful only in targeting VSDs in cell ${ }^{23-25}$ or neuronal cultures, ${ }^{26}$ and required high expression levels of genetically encoded protein targeting modalities, VoLDeMo probes show high targeting selectivity in native brain tissue of rodent brain slices and whole Drosophila brains using endogenous membrane proteins at physiological expression levels. This can be advantageous especially in genetic models of brain disorders, which often require multiple genetic modifications ${ }^{63}$ making further manipulation in order to install genetically encoded voltage indicators (GEVIs) ${ }^{15}$ potentially difficult. GEVIs also often need to be overexpressed to provide sufficient photon output, which leads to a variety of issues including toxicity or increased membrane capacitance. ${ }^{16} \mathrm{~A}$ recent approach addressed the photon budget issues via a chemogenetic approach, attaching a synthetic fluorophore to a membrane voltage sensing protein domain. ${ }^{64}$ We describe a chemical targeting approach where the entire VSD is delivered to the cells of interest.

Remarkably, the level of selectivity observed with VoLDeMo in the striatum is comparable to the small molecule, highly hydrophilic FFN102 probe, and to the genetically encoded GFP labeling under the control of the TH promoter and qualitatively superior to DAT immunohistochemical labeling. Although the binding selectivity of VoLDeMo probes is governed by a high affinity dichloropane ligand, the dextran polymer carrier 
plays a crucial role in the targeting process. The widely used PEG linker did not perform well in these studies and led to high intracellular labeling background. The high polarity of the dextran polymer (unlike PEG) compensates for the lipophilicity of the VSD, while its flexibility (and to some extent amphiphilic character) likely enables dynamic encapsulation of the dye, leading to low background staining and high diffusion through the brain tissue. Such dynamic behavior will be studied further to optimize the targeting capacity and tissue diffusion of small polymers.

Synthetic chemistry and molecular design can also directly address the key issues related to the sensor dyes, such as photon output, ${ }^{17,64}$ sensing parameters, ${ }^{65,66}$ phototoxicity, pharmacological off-targets, and targeting properties. Future studies will focus on detailed examination of membrane depolarization events in axons, near and at the release sites including active and silent synapses. ${ }^{31}$ These efforts will guide the future optimization of the sensor, linker, and dextran polymer carrier.

The modular design of dextran-based VoLDeMo offers many possibilities in terms of the potential applications and impact. Regarding the payload, we first focused on VSDs since these sensors report on a fundamental process in the nervous system - membrane potential changes - and are exceptionally challenging to deliver to specific cells and neurites due to their high lipophilicity. In this sense, we selected a high bar for testing the targeting efficacy of the designed molecular delivery constructs. The promising results shown here indicate that the dextran platform promises a means to accommodate a wide variety of lipophilic cargo molecules. Imaging modalities such as photoacoustic (PA) imaging ${ }^{20-22}$ and short-wave infrared (SWIR) fluorescence imaging ${ }^{18,19}$ also utilize lipophilic synthetic imaging agents, and no genetically encoded PA or SWIR probes have been developed to date. The concept of the VoLDeMo targeting platform may therefore offer a promising solution to cell specific targeting of PA and SWIR probes in the brain. Our platform may also be applied to include actuators, signaling modulators, and drugs, thus unlocking chemical and chemo-genetic approaches to cell specific imaging and pharmacology. ${ }^{67}$ 
In terms of the cell-targeting component of VoLDeMo probes, the dichloropane can be substituted for a variety of ligands, unlocking the possibilities of targeting phenotype/pathology-associated cell surface molecules.

Furthermore, the VoLDeMo system will be readily adaptable to chemo-genetic targeting using covalent tagging strategies (e.g. SNAP-tag ${ }^{68}$, Halo-tag $^{69}$, TMP-tag $^{70}$ or Spy-tag $^{71}$ ) by substituting the dichloropane ligand for a suitable electrophile. The incubation concentrations used with VoLDeMo ( $100 \mathrm{nM})$ are in the range required for the current version of Halo-tag.

Balancing the noted advantages and exciting potentialities of the chemical probes, there are a number of challenges associated with chemical targeting and application of exogenous reagents that need to be addressed in future research. In the current version of the VoLDeMo system, targeting DAT or NET by the dichloropane ligand perturbs the experimental system by blocking these native neurotransmitter transporters. This is a general problem with receptor ligands, as the agonist/antagonist activity of these compounds perturbs the system under study. We envision tackling this issue by employing a traceless labeling approach, recently exemplified by tagging AMPA receptors with a fluorophore in brain tissue without functional consequences for the receptor. ${ }^{72}$ Alternatively, targeting ligands (e.g., small molecules, peptides, and nanobodies) that do not perturb the function of the targeted molecule/receptor may be used. We expect a proliferation of such targeting ligands in the next decade for a wide range of molecular targets expressed in defined cell types and cell phenotypes. For in vivo applications, another major issue that arises is the mode of delivery of exogenous diffusible chemical probes to the brain tissue. In small laboratory animals, local injection into brain parenchyma is a feasible option. ${ }^{32}$ However, in the long term, less invasive methods will be required for research in animals, and to pave the way toward clinical applications - one of the major rationales for investing effort and resources into the development of chemically targeted imaging and pharmacological agents. These may include intrathecal administration that circumvents the blood-brain barrier, or intravenous administration coupled with blood-brainbarrier (BBB) penetration approaches, based on either temporary physical disruption of BBB (e.g., focused ultrasound methods) or exploitation of native transporter systems. In anticipation of major advances in the non- 
invasive brain delivery modalities, we believe the exploration and development of cell- and molecule-specific targeting systems is imperative.

In summary, VoLDeMo probes represent the first non-genetic platform for targeting lipophilic voltage sensitive dyes to specific neuronal cells and their axons in brain tissue. Although many issues remain to be addressed in terms of functional parameters and practical applicability of targeted VSDs, as outlined above, small carbohydrate polymers show great promise as the molecular platform for cell-selective imaging and pharmacology. We have demonstrated the feasibility of a chemical targeting approach that will be applicable to other lipophilic sensors, actuators or drugs. 


\section{Methods}

All experimental procedures are described in detail in the Supplementary Information.

General procedure for synthesis of azidopropyl-carbamate dextran(6000) (7-w). Dried dextran(6000) (1.0 eq.) and dried $\mathrm{LiCl}\left(1.0 \mathrm{mg} / \mathrm{mg}\right.$ dextran) were dissolved in dry DMF $\left(20 \mu \mathrm{L} / \mathrm{mg}\right.$ dextran) at $70{ }^{\circ} \mathrm{C}$ under $\mathrm{Ar}$ atmosphere (dissolution was completed within $10 \mathrm{~min})$. Dry DIPEA (1.0 $\mu \mathrm{L} / \mathrm{mg}$ dextran) was added, followed by addition of $\mathrm{N}$-(3-azidopropyl)-O-(4-nitrophenyl) carbamate (6,1.1 eq. with respect to expected coverage) resulting in immediate change of color from colorless to yellow. The resulting solution was stirred at $70{ }^{\circ} \mathrm{C}$ for $24 \mathrm{~h}$. After cooling to r.t., volatiles were removed by rotary evaporation $\left(40^{\circ} \mathrm{C}\right)$. The resulting thick yellowbrown oil was dissolved in $\mathrm{H}_{2} \mathrm{O}(2.0 \mathrm{~mL})$ and dialyzed against $\mathrm{H}_{2} \mathrm{O}$ (Slide-A-Lyzer G2, Thermo Fisher, MWCO $2000 \mathrm{Da}$ ) for 3 days with $2 \times$ per day exchange of dialysate for fresh $\mathrm{H}_{2} \mathrm{O}$. Removing the aqueous solvent by rotary evaporation $\left(40{ }^{\circ} \mathrm{C}\right)$ provided azidopropyl carbamate dextran $(7-w)$ as a white solid. Number $w$ in $7-w$ designates the average number of azidopropyl groups per 6000 Da dextran polymer molecule as determined by ${ }^{1} \mathrm{H}$ NMR (see Supplementary Information for details). The $\mathrm{N}_{3}$ coverage yield and the total yield were highly reproducible for a range of dextran-6 ratios ( $84-87 \%$ and $87-90 \%$ respectively). IR (ATR, diamond) showed a band at $2103 \mathrm{~cm}^{-1}$ corresponding to the $\mathrm{N}_{3}$ asymmetric stretch.

Synthesis of di-2-ANEP-VoLDeMo (11). To 7-3.25 (6.3 mg, $3.2 \mu \mathrm{mol}_{\text {of }} \mathrm{N}_{3}, 1.0$ eq. $\left.\mathrm{N}_{3}\right)$ in a $1 \mathrm{~mL}$ V-shaped vial with a stir bar was added di-2-ANEPB-BCN $(8,73.9 \mu \mathrm{L}$ of a $10 \mathrm{mg} / \mathrm{mL}$ sol. in DMSO, $0.74 \mathrm{mg}, 1.2 \mu \mathrm{mol}$, $1.1 \times 1 / 3$ eq. per $\mathrm{N}_{3}$ group) and dichloropane-BCN $(\mathbf{1 0}, 170.8 \mu \mathrm{L}$ of a $7.7 \mathrm{mg} / \mathrm{mL}$ sol. in DMSO, $1.3 \mathrm{mg}, 2.3 \mu \mathrm{mol}$, $1.1 \times 2 / 3$ eq. per $\mathrm{N}_{3}$ group). The resulting solution was stirred at r.t. for $48 \mathrm{~h}$. After diluting with $\mathrm{H}_{2} \mathrm{O}(2.0 \mathrm{~mL})$, the crude solution was dialyzed against $\mathrm{H}_{2} \mathrm{O}$ (Slide-A-Lyzer G2, Thermo Fisher, MWCO 3500 Da) for 3 days with 2× per day exchange of dialysate for fresh $\mathrm{H}_{2} \mathrm{O}$. Post-dialysis, the membrane remained red indicating some extent of non-specific binding of ANEP to the dialysis membrane. Removing the aqueous solvent by rotary evaporation $\left(40{ }^{\circ} \mathrm{C}\right)$ provided di-2-ANEP-VoLDeMo (11) as a red solid. Yield: $6.3 \mathrm{mg}(77 \%)$. No signal at $2103 \mathrm{~cm}^{-1}$ was observed in the IR (ATR, diamond) spectrum of $\mathbf{1 1}$ which would correspond to the $\mathrm{N}_{3}$ 
asymmetric stretch of the parent 7-3.25. di-2-ANEP-VoLDeMo (11) was obtained with a coverage of 0.74 ANEP/polymer and 1.38 ligand/polymer as determined by ${ }^{1} \mathrm{H}$ NMR (Supplementary Fig. S2).

Synthesis of dsRVF5-VoLDeMo (12). To 7-2.15 (5.1 mg, $1.7 \mu \mathrm{mol}$ of $\mathrm{N}_{3}, 1.0$ eq. $\left.\mathrm{N}_{3}\right)$ in a $1 \mathrm{~mL}$ V-shaped vial with a stir bar was added dsRVF5-BCN $(9,76.7 \mu \mathrm{L}$ of a $10 \mathrm{mg} / \mathrm{mL}$ sol. in DMSO, $0.77 \mathrm{mg}, 0.63 \mu \mathrm{mol}$, $1.1 \times 1 / 3$ eq. per $\mathrm{N}_{3}$ group) and dichloropane-BCN $(\mathbf{1 0}, 94.6 \mu \mathrm{L}$ of a $7.7 \mathrm{mg} / \mathrm{mL}$ sol. in DMSO, $0.73 \mathrm{mg}, 1.3 \mu \mathrm{mol}$, $1.1 \times 2 / 3$ eq. per $\mathrm{N}_{3}$ group). The resulting solution was stirred at r.t. for $48 \mathrm{~h}$. After diluting with $\mathrm{H}_{2} \mathrm{O}(2.0 \mathrm{~mL})$, the crude solution was dialyzed against $\mathrm{H}_{2} \mathrm{O}$ (Slide-A-Lyzer G2, Thermo Fisher, MWCO 3500 Da) for 3 days with $2 \times$ per day exchange of dialysate for fresh $\mathrm{H}_{2} \mathrm{O}$. The membrane was colorless post-dialysis, indicating negligible non-specific binding of dsRVF5 to the dialysis membrane (unlike ANEP, vide supra). Removing the aqueous solvent by rotary evaporation $\left(40^{\circ} \mathrm{C}\right)$ provided dsRVF5-VoLDeMo (12) as a red solid. Yield: $5.9 \mathrm{mg}$ (91\%). No signal at $2103 \mathrm{~cm}^{-1}$ was observed in the IR (ATR, diamond) spectrum of 12 which would correspond to the $\mathrm{N}_{3}$ asymmetric stretch of the parent 7-2.15. dsRVF5-VoLDeMo (12) was obtained with a coverage of 0.64 ANEP/polymer and 1.33 ligand/polymer as determined by ${ }^{1} \mathrm{H}$ NMR (Supplementary Fig. S3).

\section{Acknowledgements}

This paper is dedicated to the memory of James H. Handelman, a visionary and supporter of fundamental science. We thank Matthew Ding and Eva Fialová (both Department of Chemistry, Columbia University) for assistance with compound synthesis. We also thank Dr. Brandon Fowler (Department of Chemistry, Columbia University) for HRMS analysis. T.F. is the recipient of the Alfred Bader Fellowship in Organic Chemistry. This work was supported by the G. Harold \& Leila Y. Mathers Charitable Foundation (to D. Sames) and the Collaborative and Multidisciplinary Pilot Research (CaMPR) award (to D.M.M.). The imaging work in the groups of D. Sames and D. Sulzer is also supported by the National Institute of Mental Health, NIMH (R01MH108186). D. Sulzer's work is funded by NIDA R0107418 and the JPB and Parkinson's Foundations. The work in Drosophila brains was supported by John F. and Nancy A. Emmerling Fund of The Pittsburgh 
Foundation (to Z.F.), start-up funds from the University of Pittsburgh Department of Psychiatry (to Z.F.), startup funds from the University of Pittsburgh Department of Neurobiology (to C.E.C), and funding from the National Institutes of Health: R21AG059386 (to M.J.P.), R01GM108073 (to M.J.P.), and R21NS095614 (to M.J.P.). Y.B. and R.Y. are supported by the NEI (DP1EY024503, R01EY011787) and NIMH (R01MH101218, R01MH100561) and in part by the U. S. Army Research Laboratory and the U. S. Army Research Office under contract number W911NF-12-1-0594 (MURI).

\section{Author contributions}

D. Sames and D.M.M. conceptualized the project. T.F., J.W. and D. Sames designed the research with assistance from P.Š. T.F., J.W. and D. Sames wrote the manuscript. T.F., P. ̌̌. and E.C.N. synthesized the compounds. J.W. and M.D. carried out experiments in HEK cell cultures and mouse acute brain slice. J.W. and Y.B. carried out electrophysiological experiments with consultations from R.Y. Experiments in Drosophila brains were conducted by C.E.C., K.J.F. and Z.F with consultations from M.J.P. The project was overseen by D. Sames, D. Sulzer and Z.F. All authors edited the manuscript.

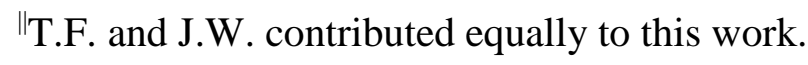

\section{Competing interests}

The authors declare no competing financial interest.

\section{ORCID iD}

T.F. 0000-0001-6949-4561, J.W. 0000-0002-2188-3629, P.Š. 0000-0003-0317-0630, C.E.C. 0000-0002-40381501, Z.F. 0000-0001-6460-0118. 


\section{References}

1. Sakmann, B. \& Neher, E. Patch Clamp Techniques for Studying Ionic Channels in Excitable Membranes. Annual Review of Physiology 46, 455-472 (1984).

2. Segev, A., Garcia-Oscos, F. \& Kourrich, S. Whole-cell Patch-clamp Recordings in Brain Slices. JoVE (Journal of Visualized Experiments) e54024-e54024 (2016). doi:10.3791/54024

3. Tsien, R. Y. A non-disruptive technique for loading calcium buffers and indicators into cells. Nature 290, 527-528 (1981).

4. Grynkiewicz, G., Poenie, M. \& Tsien, R. Y. A new generation of Ca2+ indicators with greatly improved fluorescence properties. J. Biol. Chem. 260, 3440-3450 (1985).

5. Cohen, L. B. \& Salzberg, B. M. Optical measurement of membrane potential. in Reviews of Physiology, Biochemistry and Pharmacology, Volume 83 35-88 (Springer, Berlin, Heidelberg, 1978). doi:10.1007/3-54008907-1_2

6. Peterka, D. S., Takahashi, H. \& Yuste, R. Imaging Voltage in Neurons. Neuron 69, 9-21 (2011).

7. Loew, L. M. Design and Use of Organic Voltage Sensitive Dyes. in Membrane Potential Imaging in the Nervous System and Heart 27-53 (Springer, Cham, 2015). doi:10.1007/978-3-319-17641-3_2

8. Miller, E. W. Small molecule fluorescent voltage indicators for studying membrane potential. Current Opinion in Chemical Biology 33, 74-80 (2016).

9. Grinvald, A. \& Hildesheim, R. VSDI: a new era in functional imaging of cortical dynamics. Nat Rev Neurosci 5, 874-885 (2004).

10. Kuhn, B., Denk, W. \& Bruno, R. M. In vivo two-photon voltage-sensitive dye imaging reveals top-down control of cortical layers 1 and 2 during wakefulness. PNAS 105, 7588-7593 (2008).

11. Airan, R. D. et al. High-Speed Imaging Reveals Neurophysiological Links to Behavior in an Animal Model of Depression. Science 317, 819-823 (2007). 
12. Storace, D. et al. Genetically Encoded Protein Sensors of Membrane Potential. in Membrane Potential Imaging in the Nervous System and Heart 493-509 (Springer, Cham, 2015). doi:10.1007/978-3-319-176413_20

13. Sepehri Rad, M. et al. Voltage and Calcium Imaging of Brain Activity. Biophysical Journal 113, 2160 2167 (2017).

14. Xu, Y., Zou, P. \& Cohen, A. E. Voltage imaging with genetically encoded indicators. Current Opinion in Chemical Biology 39, 1-10 (2017).

15. Platisa, J. \& Pieribone, V. A. Genetically encoded fluorescent voltage indicators: are we there yet? Current Opinion in Neurobiology 50, 146-153 (2018).

16. Kulkarni, R. U. \& Miller, E. W. Voltage Imaging: Pitfalls and Potential. Biochemistry 56, 5171-5177 (2017).

17. Lavis, L. D. Chemistry Is Dead. Long Live Chemistry! Biochemistry 56, 5165-5170 (2017).

18. Treger, J. S., Priest, M. F., Iezzi, R. \& Bezanilla, F. Real-Time Imaging of Electrical Signals with an Infrared FDA-Approved Dye. Biophysical Journal 107, L09-L12 (2014).

19. Carr, J. A. et al. Shortwave infrared fluorescence imaging with the clinically approved near-infrared dye indocyanine green. PNAS 115, 4465-4470 (2018).

20. Weber, J., Beard, P. C. \& Bohndiek, S. E. Contrast agents for molecular photoacoustic imaging. Nature Methods 13, 639-650 (2016).

21. Zhang, H. K. et al. Listening to membrane potential: photoacoustic voltage-sensitive dye recording. JBO, JBOPFO 22, 045006 (2017).

22. Rao, B., Zhang, R., Li, L., Shao, J.-Y. \& Wang, L. V. Photoacoustic imaging of voltage responses beyond the optical diffusion limit. Scientific Reports 7, 2560 (2017).

23. Hinner, M. J., Hübener, G. \& Fromherz, P. Enzyme-Induced Staining of Biomembranes with VoltageSensitive Fluorescent Dyes. J. Phys. Chem. B 108, 2445-2453 (2004). 
24. Hinner, M. J., Hübener, G. \& Fromherz, P. Genetic Targeting of Individual Cells with a Voltage-

Sensitive Dye through Enzymatic Activation of Membrane Binding. ChemBioChem 7, 495-505

25. Ng, D. N. \& Fromherz, P. Genetic Targeting of a Voltage-Sensitive Dye by Enzymatic Activation of Phosphonooxymethyl-ammonium Derivative. ACS Chem. Biol. 6, 444-451 (2011).

26. Liu, P., Grenier, V., Hong, W., Muller, V. R. \& Miller, E. W. Fluorogenic Targeting of VoltageSensitive Dyes to Neurons. J. Am. Chem. Soc. 139, 17334-17340 (2017).

27. Grenier, V., Walker, A. S. \& Miller, E. W. A Small-Molecule Photoactivatable Optical Sensor of Transmembrane Potential. J. Am. Chem. Soc. 137, 10894-10897 (2015).

28. Gubernator, N. G. et al. Fluorescent False Neurotransmitters Visualize Dopamine Release from Individual Presynaptic Terminals. Science 324, 1441-1444 (2009).

29. Lee, M., Gubernator, N. G., Sulzer, D. \& Sames, D. Development of pH-responsive fluorescent false neurotransmitters. J. Am. Chem. Soc. 132, 8828-8830 (2010).

30. Rodriguez, P. C. et al. Fluorescent dopamine tracer resolves individual dopaminergic synapses and their activity in the brain. PNAS 110, 870-875 (2013).

31. Pereira, D. B. et al. Fluorescent false neurotransmitter reveals functionally silent dopamine vesicle clusters in the striatum. Nature Neuroscience 19, 578-586 (2016).

32. Dunn, M. et al. Designing a norepinephrine optical tracer for imaging individual noradrenergic synapses and their activity in vivo. Nature Communications 9, 2838 (2018).

33. Merchant, P., Sulzer, D. \& Sames, D. Synaptic optical imaging platforms: Examining pharmacological modulation of neurotransmitter release at discrete synapses. Neuropharmacology 98, 90-94 (2015).

34. Antic, S. \& Zecevic, D. Optical signals from neurons with internally applied voltage-sensitive dyes. $J$. Neurosci. 15, 1392-1405 (1995).

35. Tsau, Y. et al. Dye screening and signal-to-noise ratio for retrogradely transported voltage-sensitive dyes. Journal of Neuroscience Methods 70, 121-129 (1996). 
36. Kulkarni, R. U. et al. A Rationally Designed, General Strategy for Membrane Orientation of Photoinduced Electron Transfer-Based Voltage-Sensitive Dyes. ACS Chem. Biol. 12, 407-413 (2017).

37. Kulkarni, R. U. et al. Voltage-sensitive rhodol with enhanced two-photon brightness. PNAS 114, $2813-$ 2818 (2017).

38. Meltzer, P. C., Liang, A. Y., Brownell, A. L., Elmaleh, D. R. \& Madras, B. K. Substituted 3phenyltropane analogs of cocaine: synthesis, inhibition of binding at cocaine recognition sites, and positron emission tomography imaging. J. Med. Chem. 36, 855-862 (1993).

39. Carroll, F. I. et al. Synthesis, Ligand Binding, and QSAR (CoMFA and Classical) Study of 3.beta.-(3'Substituted phenyl)-, 3.beta.-(4'-Substituted phenyl)-, and 3.beta.-(3',4'-Disubstituted phenyl)tropane2.beta.-carboxylic Acid Methyl Esters. J. Med. Chem. 37, 2865-2873 (1994).

40. Wang, K. H., Penmatsa, A. \& Gouaux, E. Neurotransmitter and psychostimulant recognition by the dopamine transporter. Nature 521, 322-327 (2015).

41. Cha, J. H. et al. Rhodamine-Labeled $2 \beta$-Carbomethoxy-3 $\beta$-(3,4-dichlorophenyl)tropane Analogues as High-Affinity Fluorescent Probes for the Dopamine Transporter. J. Med. Chem. 48, 7513-7516 (2005).

42. Karpowicz, R. J., Dunn, M., Sulzer, D. \& Sames, D. APP+, a Fluorescent Analogue of the Neurotoxin MPP+, Is a Marker of Catecholamine Neurons in Brain Tissue, but Not a Fluorescent False Neurotransmitter. ACS Chem. Neurosci. 4, 858-869 (2013).

43. Eriksen, J. et al. Visualization of Dopamine Transporter Trafficking in Live Neurons by Use of Fluorescent Cocaine Analogs. J. Neurosci. 29, 6794-6808 (2009).

44. Brogden, R. N., Heel, R. C., Speight, T. M. \& Avery, G. S. Nomifensine: A Review of its Pharmacological Properties and Therapeutic Efficacy in Depressive Illness. Drugs 18, 1-24 (1979).

45. Dupayage, L., Nouvel, C. \& Six, J.-L. Protected versus unprotected dextran macroinitiators for ATRP synthesis of Dex-g-PMMA. J. Polym. Sci. A Polym. Chem. 49, 35-46 (2011).

46. $\mathrm{Wu}$, J. et al. Binding characteristics between polyethylene glycol (PEG) and proteins in aqueous solution. J. Mater. Chem. B 2, 2983-2992 (2014). 
47. Prifti, E. et al. A Fluorogenic Probe for SNAP-Tagged Plasma Membrane Proteins Based on the Solvatochromic Molecule Nile Red. ACS Chem. Biol. 9, 606-612 (2014).

48. Cheng, H. N. \& Neiss, T. G. Solution NMR Spectroscopy of Food Polysaccharides. Polymer Reviews 52, 81-114 (2012).

49. Vercelli, A., Repici, M., Garbossa, D. \& Grimaldi, A. Recent techniques for tracing pathways in the central nervous system of developing and adult mammals. Brain Research Bulletin 51, 11-28 (2000).

50. Syková, E. \& Nicholson, C. Diffusion in Brain Extracellular Space. Physiological Reviews 88, 12771340 (2008).

51. Xiao, F., Nicholson, C., Hrabe, J. \& Hrabětová, S. Diffusion of Flexible Random-Coil Dextran Polymers Measured in Anisotropic Brain Extracellular Space by Integrative Optical Imaging. Biophysical Journal 95, 1382-1392 (2008).

52. Fluhler, E., Burnham, V. G. \& Loew, L. M. Spectra, membrane binding, and potentiometric responses of new charge shift probes. Biochemistry 24, 5749-5755 (1985).

53. Matsuda, W. et al. Single Nigrostriatal Dopaminergic Neurons Form Widely Spread and Highly Dense Axonal Arborizations in the Neostriatum. J. Neurosci. 29, 444-453 (2009).

54. Lein, E. S. et al. Genome-wide atlas of gene expression in the adult mouse brain. Nature 445, 168-176 (2007).

55. Interactive Atlas Viewer :: Atlas Viewer. Available at: http://atlas.brainmap.org/atlas atlas $=2 \#$ atlas $=2 \&$ plate $=100884129 \&$ structure $=549 \& \mathrm{x}=7799.908447265625 \& \mathrm{y}=4023.348426$ 8188477\&zoom=-3\&resolution=16.11\&z=5. (Accessed: 7 th August 2018)

56. Sara, S. J. Locus Coeruleus in time with the making of memories. Current Opinion in Neurobiology 35, 87-94 (2015).

57. Fujimori, K., Covell, D. G., Fletcher, J. E. \& Weinstein, J. N. A Modeling Analysis of Monoclonal Antibody Percolation Through Tumors: A Binding-Site Barrier. J Nucl Med 31, 1191-1198 (1990). 
58. Freyberg, Z. et al. Mechanisms of amphetamine action illuminated through optical monitoring of dopamine synaptic vesicles in Drosophila brain. Nature Communications 7, 10652 (2016).

59. Aguilar, J. I. et al. Neuronal Depolarization Drives Increased Dopamine Synaptic Vesicle Loading via VGLUT. Neuron 95, 1074-1088.e7 (2017).

60. Cao, G. et al. Genetically Targeted Optical Electrophysiology in Intact Neural Circuits. Cell 154, 904913 (2013).

61. Schoofs, A., Hückesfeld, S. \& Pankratz, M. J. Serotonergic network in the subesophageal zone modulates the motor pattern for food intake in Drosophila. Journal of Insect Physiology 106, 36-46 (2018).

62. Pörzgen, P., Park, S. K., Hirsh, J., Sonders, M. S. \& Amara, S. G. The Antidepressant-Sensitive Dopamine Transporter inDrosophila melanogaster: A Primordial Carrier for Catecholamines. Mol Pharmacol 59, 83-95 (2001).

63. Trancikova, A., Ramonet, D. \& Moore, D. J. Chapter 11 - Genetic Mouse Models of Neurodegenerative Diseases. in Progress in Molecular Biology and Translational Science (eds. Chang, K. T. \& Min, K.-T.) 100, 419-482 (Academic Press, 2011).

64. Schreiter, E. R., Lavis, L. D. \& Abdelfattah, A. Chemigenetic Voltage Indicators. WO2018102577 (A1) (2018).

65. Fromherz, P., Hübener, G., Kuhn, B. \& Hinner, M. J. ANNINE-6plus, a voltage-sensitive dye with good solubility, strong membrane binding and high sensitivity. Eur Biophys J 37, 509-514 (2008).

66. Deal, P. E., Kulkarni, R. U., Al-Abdullatif, S. H. \& Miller, E. W. Isomerically Pure Tetramethylrhodamine Voltage Reporters. J. Am. Chem. Soc. 138, 9085-9088 (2016).

67. Shields, B. C. et al. Deconstructing behavioral neuropharmacology with cellular specificity. Science 356, eaaj2161 (2017).

68. Mollwitz, B. et al. Directed Evolution of the Suicide Protein O6-Alkylguanine-DNA Alkyltransferase for Increased Reactivity Results in an Alkylated Protein with Exceptional Stability. Biochemistry 51, 986994 (2012). 
69. Los, G. V. et al. HaloTag: A Novel Protein Labeling Technology for Cell Imaging and Protein Analysis. ACS Chem. Biol. 3, 373-382 (2008).

70. Chen, Z., Jing, C., Gallagher, S. S., Sheetz, M. P. \& Cornish, V. W. Second-Generation Covalent TMPTag for Live Cell Imaging. J. Am. Chem. Soc. 134, 13692-13699 (2012).

71. Zakeri, B. et al. Peptide tag forming a rapid covalent bond to a protein, through engineering a bacterial adhesin. PNAS 109, E690-E697 (2012).

72. Wakayama, S. et al. Chemical labelling for visualizing native AMPA receptors in live neurons. Nature Communications 8, 14850 (2017). 